\title{
Safety Report
}

National Cancer Institute

\section{Source}

National Cancer Institute. Safety Report. NCI Thesaurus. Code C93488.

A report that provides notification of an adverse event, product problem, and/or

information that is relevant to either. A report typically includes causal association,

management strategies, authorship, sender/receiver organizations, subject of adverse event, or name of product. 